\title{
Statistical Location Model for Abdominal Organ Localization
}

\author{
Jianhua Yao and Ronald M. Summers \\ Imaging Biomarkers and Computer Aided Diagnosis Lab, Clinical Center, \\ The National Institutes of Health, Bethesda, MD 20892, USA
}

\begin{abstract}
Initial placement of the models is an essential pre-processing step for model-based organ segmentation. Based on the observation that organs move along with the spine and their relative locations remain relatively stable, we built a statistical location model (SLM) and applied it to abdominal organ localization. The model is a point distribution model which learns the pattern of variability of organ locations relative to the spinal column from a training set of normal individuals. The localization is achieved in three stages: spine alignment, model optimization and location refinement. The SLM is optimized through maximum a posteriori estimation of a probabilistic density model constructed for each organ. Our model includes five organs: liver, left kidney, right kidney, spleen and pancreas. We validated our method on 12 abdominal CTs using leave-one-out experiments. The SLM enabled reduction in the overall localization error from $62.0 \pm 28.5 \mathrm{~mm}$ to $5.8 \pm 1.5 \mathrm{~mm}$. Experiments showed that the SLM was robust to the reference model selection.
\end{abstract}

\section{Introduction}

Segmentation of anatomical structures is often the first step in computer-aided diagnosis using medical images. In recent years there has been considerable interest in methods that use deformable models or atlases to segment anatomical structures. One category of deformable models, such as active contour models [1] and geodesic level sets [2], is based on the optimization of objective functions. Another category of models, including active shape models (ASM) [3], probabilistic atlases [4], and statistical shape models [5], is constructed based on prior information extracted from samples. The motivation is to achieve robust segmentation by constraining solutions to be valid examples of the structure modeled in a population.

Abdominal organ segmentation on CT scans is a challenging task due to the following reasons. First, different organs have similar Hounsfield number, which limits the use of thresholding methods. Second, organs have irregular shapes and often demonstrate large anatomical variations amongst individuals. Third, image artifacts, such as beam-hardening, partial-volume and motion, raise more difficulties.

Instead of segmenting each organ separately, multi-organ segmentation has attracted investigations in recent years [4-6]. Park et al. [4] constructed a probabilistic atlas of three organs (liver, both kidneys) and the spinal cord. The atlas was optimized in a Bayesian framework using mutual information as the similarity measure. Okada 

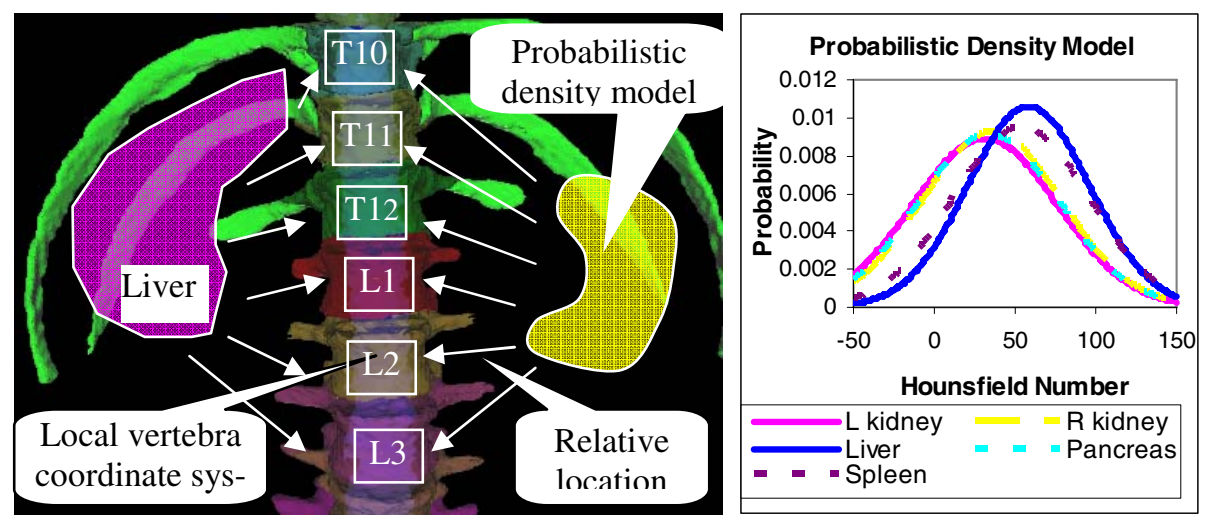

Fig. 1. Organ location model. Left: relative location model; right: probabilistic density model.

et al. [5] proposed a hierarchical statistical atlas to embed the constraints on interrelations amongst organs in an orga nization of probabilistic atlases and statistical shape models. Shimizu [6] et al. proposed a simultaneous extraction method for 12 abdominal organs based on abdominal cavity standardization and the EM algorithm.

Initial placement of the models is essential to the success of model-based methods, and this is especially important for organ segmentation due to the large variability. Most methods [4] [7] require the models be placed in close proximity of the targets. Some methods relied on human experts for manual initialization and guidance [4]. Fujimoto et al. [7] set up a coordinate system centered at the T12 vertebra and employed normalized distance for model initialization.

In this paper, we propose a novel method to initialize the model location for multiorgan segmentation. We observed that the spinal column supports the human upper body. When the spine moves, the organs move along with it and the relative location between the organs and the spine remains relatively stable. Furthermore, the organ location configurations amongst normal individuals are similar. Therefore, we model the statistics of the organ location relative to the spinal column using a point distribution model. The organs are also equipped with probabilistic density models. Our model includes five abdominal organs: liver, spleen, pancreas and both kidneys.

\section{Methods}

Our method is summarized as follows. In the modeling phase, we first segment the spinal column and partition it into vertebrae. The vertebrae are used as anchor points, and the relative locations between organs and vertebrae are recorded. We then build a statistical location model (SLM) to learn all possible configurations of organ relative locations from a training set of normal individuals. We also build a probabilistic density model for each organ. In the application phase, the SLM is applied to a new data set to obtain the initial organ location through maximum a posteriori (MAP) estimation of the probabilistic density model. 


\subsection{Automated Spinal Column Extraction and Partitioning}

The details of the automated spinal column extraction and partitioning can be found in [8]. A threshold of $200 \mathrm{HU}$ is applied to mask out the bone pixels. Then a connected component analysis is conducted to obtain the initial spine segmentation. The spinal cord is then extracted using a watershed algorithm and a directed acyclic graph search. Then curved planar reformation is computed along the centerline to partition the spinal column into vertebrae. After that, the ribs are detected using features such as size, location, shape, orientation and density. Finally the vertebrae are labeled on the basis of two pieces of anatomical knowledge: 1) one vertebra has at most two attached ribs; 2) ribs are attached only to thoracic vertebrae. Figure 1 illustrated one example of partitioned spinal column. In our model, only six vertebrae, T10, T11, T12, L1, L2 and L3, are included since they are present in most abdominal CT scans.

\subsection{Organ Location Model}

We build an organ location model based on the spinal column. We fit a B-Spline curve for the centerline of the spinal cord. A local frame $\left\{c\left(V_{i}\right), \vec{t}\left(V_{i}\right), \vec{f}_{1}\left(V_{i}\right), \vec{f}_{2}\left(V_{i}\right)\right\}$ is established for each vertebra $V_{i}$. Here $c\left(V_{i}\right)$ is the center point of $V_{i}$ at the spinal cord, $\vec{t}\left(V_{i}\right)$ is the tangent of the spinal cord, $\vec{f}_{1}\left(V_{i}\right)$ and $\vec{f}_{2}\left(V_{i}\right)$ are two orthogonal vectors on the plane perpendicular to $\vec{t}\left(V_{i}\right)$. Given the local frame at $V_{i}$, the relative location and orientation of each organ can be computed.

In the modeling phase, we manually segment each organ and compute its 3D surface. For an organ $O_{j}$, we use the center of mass of the 3D surface as its center. The relative location between an organ $O_{j}$ and a vertebra $V_{i}$ is defined as,

$$
r l\left(O_{j}, V_{i}\right)=c\left(O_{j}\right)-c\left(V_{i}\right)
$$

here $c\left(O_{j}\right)$ is the center of organ $O_{j}$ and $c\left(V_{i}\right)$ is the center of vertebra $V_{i}$. The location vector of organ $O_{j}$ is then defined as,

$$
L\left(O_{j}\right)=\left\{r l\left(O_{j}, V_{1}\right), \operatorname{rl}\left(O_{j}, V_{2}\right), \ldots, r l\left(O_{j}, V_{n}\right)\right\}
$$

here $n$ is the number of vertebrae in the model ( $n=6$ for vertebra T10 to L3). The location vector for multiple organs is the concatenation of the location vector of each organ in the model, i.e.,

$$
L=\left\{L\left(O_{I}\right), L\left(O_{2}\right), \ldots, L\left(O_{m}\right)\right\}=\left\{r l\left(O_{1}, V_{l}\right), r l\left(O_{1}, V_{2}\right), \ldots, r l\left(O_{m}, V_{n}\right)\right\}
$$

here $m$ is the total number of organs in the model $(m=5)$. The location vector is a 90 dimensional vector $(5 * 6 * 3=90)$. The organ location model is illustrated in Figure 1 .

\subsection{Probabilistic Density Model}

We build a probabilistic density model for each organ. The model is a conditional Gaussian model using sample mean and variance over the manually segmented organ region. The density model of an organ $O_{j}$ is represented as,

$$
G\left(O_{j}\right)=\left(\mu\left(O_{j}\right), \sigma\left(O_{j}\right)\right)
$$


where $\mu\left(O_{j}\right)$ is the mean tissue density and $\sigma^{2}\left(O_{j}\right)$ is the variance. Given this model, the conditional probability of a tissue $y_{i}$ belonging to organ $O_{j}$ is,

$$
p\left(y_{i} \mid O_{j}\right)=\frac{1}{\sqrt{2 \pi \sigma^{2}\left(O_{j}\right)}} \exp \left(\frac{-\left(y_{i}-\mu\left(O_{j}\right)\right)^{2}}{2 \sigma^{2}\left(O_{j}\right)}\right)
$$

Figure 1 (right) shows the probabilistic density models of five organs.
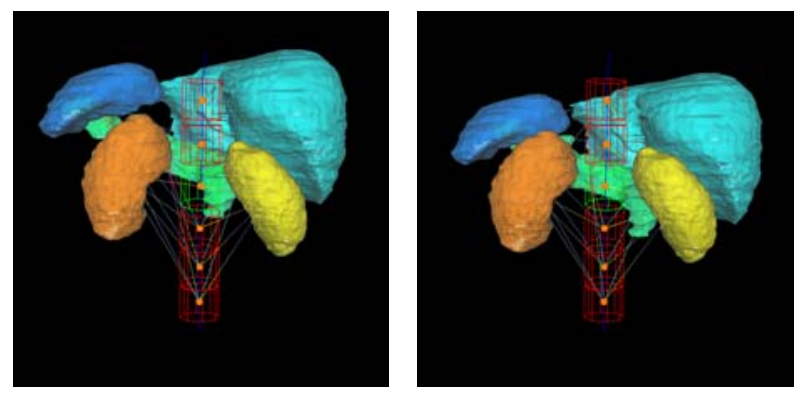

$$
-2 \sqrt{\lambda_{1}}
$$

$\boldsymbol{b}_{1}$

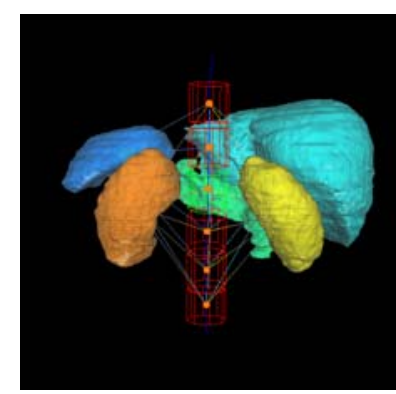

$+2 \sqrt{\lambda_{1}}$

Fig. 2. Statistical location model (SLM). Cylinders are vertebra models. Note variations in the relative locations of liver (cyan), left kidney (yellow), right kidney (orange), spleen (blue) and pancreas (green) as the largest mode $b_{1}$ is varied.

\subsection{Statistical Location Model}

Our aim is to build a statistical location model (SLM) for abdominal organs. The SLM describes both the typical location and variability of the organs from a training set of normal individuals. This is achieved by a point distribution model (PDM).

In order to compute the statistics, all training models must first be aligned. We randomly select a reference model from the training set and align other models to it. The technique is described in detail in Section 2.5.1. The alignment is through the spinal column and the aligned location models form an "allowable location domain", which can be modeled using a PDM.

A principal component analysis (PCA) method is employed to extract the "modes of variation" from the training set. Given a set of $N$ aligned location models $\left\{L_{i}\right\}$ (Eq. 3 ), the mean location model $\bar{L}$ is computed,

$$
\bar{L}=\frac{1}{N} \sum_{i=1}^{N} L_{i}
$$

A covariance matrix $S$ is constructed using the deviation from the mean model,

$$
S=\frac{1}{N} \sum_{i=1}^{N}\left(L_{i}-\bar{L}\right)\left(L_{i}-\bar{L}\right)^{T}
$$

The eigenvectors $\left\{e_{1}, e_{2}, . . e_{N}\right\}$ and their corresponding eigenvalues $\left\{\lambda_{1}, \lambda_{2}, \ldots, \lambda_{N}\right\}$ are computed. The variance explained by each eigenvector is equal to its corresponding 
eigenvalue. The first $t(t<N)$ eigenvectors are often sufficient to define the "allowable location domain". Any location model in the domain can be represented by taking the mean and adding a linear combination of the eigenvectors, i.e.,

$$
L(b)=\bar{L}+E b
$$

where $E=\left\{e_{1}, e_{2}, . . e_{t}\right\}$ is the matrix of first $t$ eigenvectors, and $b=\left(b_{l}, b_{2}, \ldots, b_{t}\right)^{\mathrm{T}}$ is the model parameter vector. By varying $b$, we can generate new location instances within the "allowable location domain". Given an instantiated location model $L(b)$, the relative location $r l\left(O_{j}, V_{i}\right)$ can be extracted from the vector (Eq. 3), and the location of an organ can be computed as,

$$
l\left(O_{j}\right)=\frac{1}{n} \sum_{i=1}^{n}\left(c\left(V_{i}\right)+r l\left(O_{j}, V_{i}\right)\right)
$$

Figure 2 shows examples of instantiated location models by varying the largest mode $b_{1}$ of the SLM.

\subsection{Organ Localization Using SLM}

Given the SLM, the organ localization is treated as an optimization problem in which we maximize the similarity between one instantiated model and an image. After the spinal column is extracted and partitioned from the image (section 2.1), the organ localization is conducted in three steps: first, the spinal column in the SLM is aligned with the spinal column in the image (2.5.1); second, the model parameter is optimized through MAP estimation of the probabilistic density model (2.5.2); and third, the location of each organ is locally refined (2.5.3).
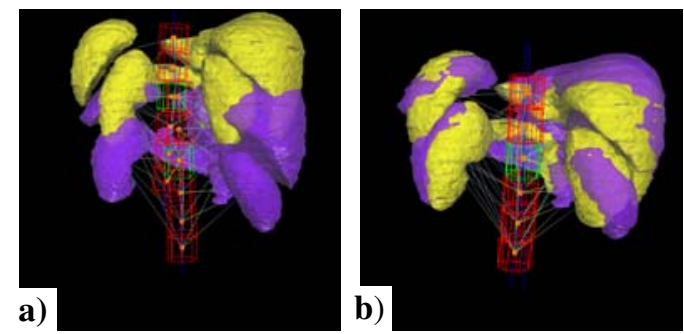

Fig. 3. Model alignment. a) Before alignment, b) after alignment

\subsubsection{Model Alignment}

The location models are aligned using the spinal column. That is, the vertebrae in one model are aligned to their corresponding vertebrae in another model. The organs are then relocated according to the spine alignment. Assuming the translation of vertebra $V_{i}$ from the moving model to the fixed model is $\Delta t\left(V_{i}\right)$, the new location of organ $O_{j}$ is,

$$
l\left(O_{j}\right)=\frac{1}{n} \sum_{i=1}^{n}\left(c\left(V_{i}\right)+\Delta t\left(V_{i}\right)+r l\left(O_{j}, V_{i}\right)\right)
$$

Currently only the positions of the vertebrae are aligned, not the orientation. Figure 3 shows two models before and after the alignment.

\subsubsection{Model Optimization}

The optimization of the SLM is through MAP estimation of the probabilistic density model. Given a location model $L(b)$, we can extract the organ location $l\left(O_{j}\right)$ using 


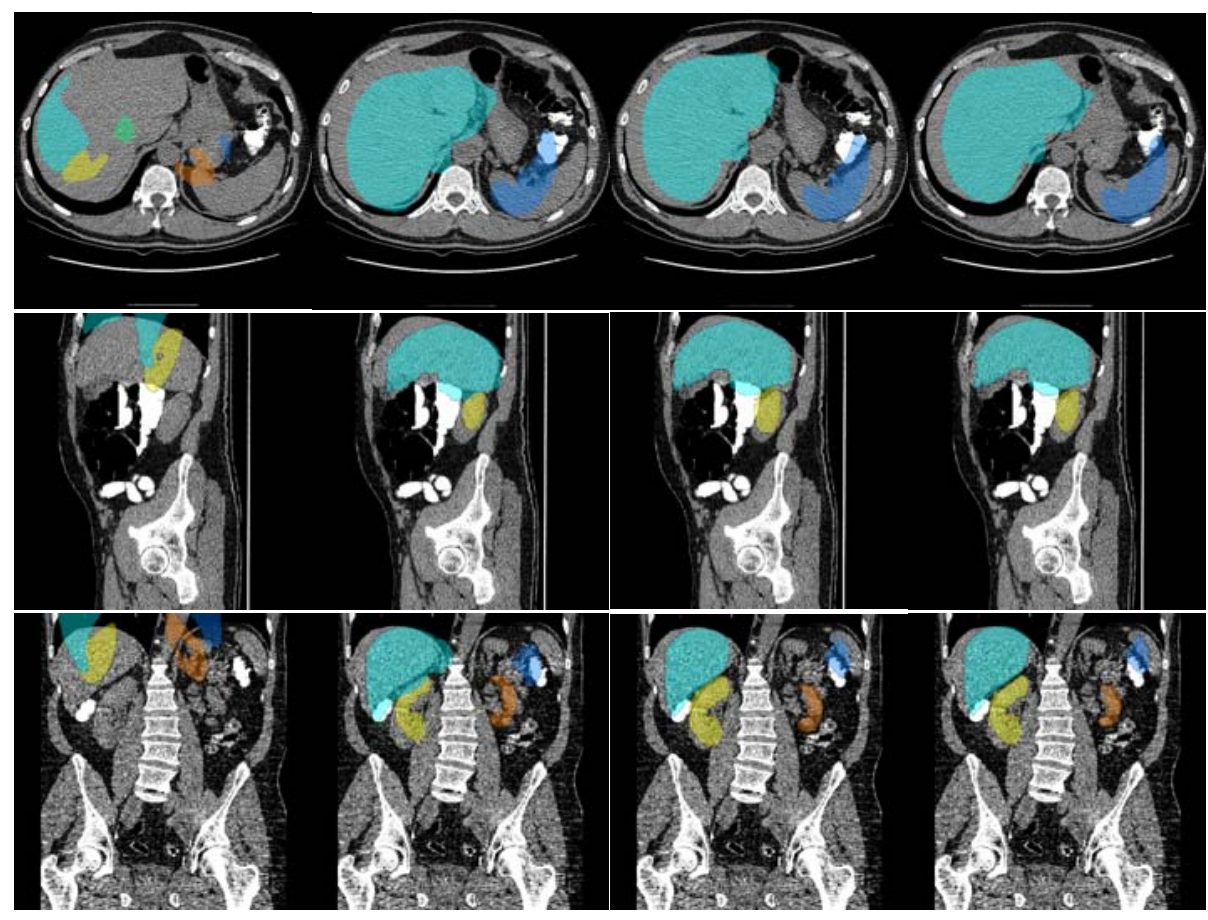

Fig. 4. Organ localization example. Reference models are superimposed on the image data, orientation not optimized. Liver (cyan), left kidney (yellow), right kidney (orange), spleen (blue) and pancreas (green).

$1^{\text {st }}$ row: axial view; $2^{\text {nd }}$ row: sagittal view; $3^{\text {rd }}$ row: coronal view

$1^{\text {st }}$ column: initial state; $2^{\text {nd }}$ column: after spine alignment; $3^{\text {rd }}$ column: after model optimization; $4^{\text {th }}$ column: after local refinement.

Eq. 3 and 9. The manually segmented organ region in the reference model is moved to location $l\left(O_{j}\right)$, and the set of voxels inside the region are generated, denoted as $\Omega\left(O_{j}\right.$, $L(b))$. The average probability that $\Omega\left(O_{j}, L(b)\right)$ coincides with organ $O_{j}$ is,

$$
P\left(\Omega\left(O_{j}, L(b)\right) \mid O_{j}\right)=\frac{1}{\left\|\Omega\left(O_{j}, L(b)\right)\right\|} \sum_{y_{i} \in \Omega\left(O_{j}, L(b)\right)} p\left(y_{i} \mid O_{j}\right)
$$

here $p\left(y_{i} \mid O_{j}\right)$ is defined in the probabilistic density model (Eq. 5). To obtain the optimal location model, we maximize the a posteriori probability for all organs. That is, the optimal model parameter $b$ is,

$$
b^{o p t}=\underset{b}{\arg \max }\left(\sum_{j=1}^{m} P\left(\Omega\left(O_{j}, L(b)\right) \mid O_{j}\right)\right)
$$

The optimization algorithm is Powell's method.

\subsubsection{Location Refinement}

Since the SLM can only model the variability present in the training set, we conduct a location refinement for each organ independently. The refinement is conducted in a 
small neighborhood of $L\left(b^{o p t}\right)$ and through MAP estimation of the probabilistic density model, i.e.,

$$
\Delta l\left(O_{j}\right)^{o p t}=\underset{\Delta l}{\arg \max }\left(P\left(\Omega\left(O_{j}, L\left(b^{o p t}\right)+\Delta l\right) \mid O_{j}\right)\right)
$$

here $\Delta l\left(O_{j}\right)^{\text {opt }}$ is the optimal location adjustment.

Figure 4 shows an example of organ localization using the statistical location model. The reference model is superimposed on the image data.

\subsection{Validation Dataset and Analysis}

Our method was validated on 12 (6 males and 6 females) abdominal CT scans. The CT reconstruction interval is $1 \mathrm{~mm}$. We manually segmented the five organs from all cases. The center of mass is treated as the location of an organ. The localization error is computed as the distance between the manually determined organ location and the computed organ location,

$$
\begin{aligned}
& \Delta d\left(O_{j}\right)=\left\|l\left(O_{j}\right)^{c p}-l\left(O_{j}\right)^{g t}\right\| \\
& \Delta d=\frac{1}{5} \sum_{j=1}^{5} \Delta d\left(O_{j}\right)
\end{aligned}
$$

here $l\left(O_{j}\right)^{c p}$ is the computed organ location (Eq. 10), $l\left(O_{j}\right)^{g t}$ is the location determined by manual segmentation (used as ground truth), $\Delta d$ is the overall localization error.

In our validation, we adopted a leave-one-out strategy. That is, we used 11 data sets to build a SLM, and applied the SLM on the left-out data to compute the organ locations.

\section{Results}

The eigenvalues of the SLM built from 12 data sets are shown in Table 1. It shows that the first 6 modes of the SLM cover more than $95 \%$ of the variability.

Table 1. Eigenvalues of the SLM

\begin{tabular}{|l|l|l|l|l|l|l|l|l|l|l|l|}
\hline$\lambda_{1}{ }^{\prime}$ & $\lambda_{2}{ }^{\prime}$ & $\lambda_{3}{ }^{\prime}$ & $\lambda_{4}{ }^{\prime}$ & $\lambda_{5}{ }^{\prime}$ & $\lambda_{6}{ }^{\prime}$ & $\lambda_{7}{ }^{\prime}$ & $\lambda_{8}{ }^{\prime}$ & $\lambda_{9}{ }^{\prime}$ & $\lambda_{10}{ }^{\prime}$ & $\lambda_{11}{ }^{\prime}$ & $\lambda_{12}{ }^{\prime}$ \\
\hline $42.6 \%$ & $27.1 \%$ & $10.0 \%$ & $7.5 \%$ & $5.9 \%$ & $2.5 \%$ & $1.9 \%$ & $1.1 \%$ & $0.7 \%$ & $0.3 \%$ & $0.1 \%$ & $0 \%$ \\
\hline
\end{tabular}

$\lambda_{i}{ }^{\prime}=\lambda_{i} / \lambda_{t}, \lambda_{t}$ is the sum of all $\lambda \mathrm{s}$

Figure 5 shows the statistics (mean and standard deviation of all leave-one-out experiments) of the localization error in each stage of the organ localization. The localization error was reduced dramatically $(91 \%)$ from the initial stage to after the final refinement. Figure 6 shows the localization error of each organ. It shows that the liver is most accurately located, but the differences of localization errors amongst organs are small.

To evaluate the sensitivity to the reference model selection, we build different SLMs using different data sets for the reference models. The leave-one-out strategy was again adopted in the validation. Table 2 shows the results with different reference models. It indicates that our model is robust to the reference model selection. 
Table 2. Localization error $(\mathrm{mm})$ of different reference models

\begin{tabular}{|l|l|l|l|l|l|l|l|l|l|l|l|l|}
\hline model & 1 & 2 & 3 & 4 & 5 & 6 & 7 & 8 & 9 & 10 & 11 & 12 \\
\hline mean $(\mathrm{mm})$ & 5.9 & 5.8 & 6.3 & 6.2 & 5.9 & 5.5 & 5.8 & 5.7 & 5.6 & 5.9 & 5.6 & 5.9 \\
\hline stdev $(\mathrm{mm})$ & 1.4 & 1.0 & 1.7 & 1.6 & 1.9 & 1.3 & 1.2 & 1.6 & 1.4 & 1.5 & 1.5 & 1.5 \\
\hline
\end{tabular}

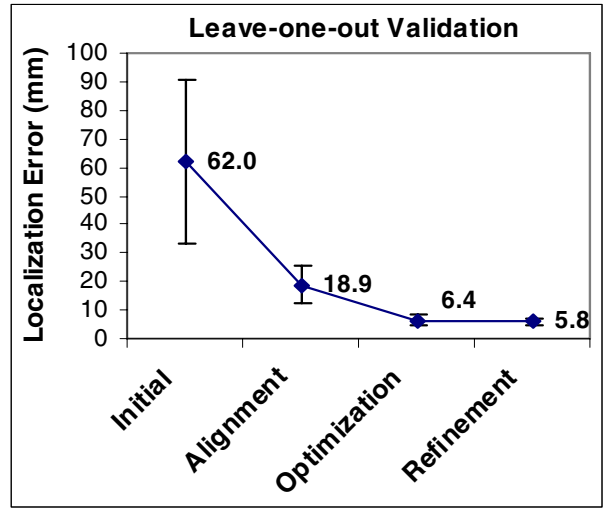

Fig. 5. Localization error in each stage of organ localization

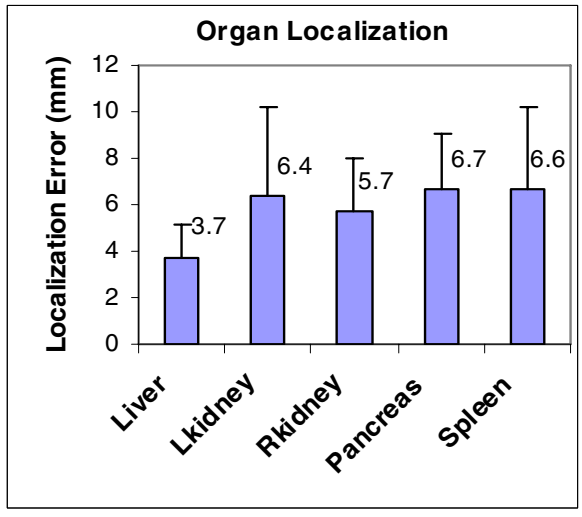

Fig. 6. Localization error for each organ

\section{Discussion}

The SLM is similar to the active shape model (ASM) [3] in the way that the model is constructed. Instead of modeling the variability of labeled landmark positions as in the ASM, we model the variability of the abdominal organ locations relative to the spinal column. Our model ensures that the instantiated organ locations are consistent with the ones in the training set. The organ localization is only the first step towards a fully automated organ segmentation method. The final segmentation can be achieved using techniques such as level sets [2], ASMs [3], and statistical atlases [5].

More work is still needed to improve the SLM. For instance, variability of organ orientation and scale can be incorporated so that the SLM can bring the model even closer to the target during the initialization. Furthermore, the constraint of relative locations amongst organs can be incorporated to prevent organs from intersecting each other. The model will be trained on a larger training set to provide better estimation of the variability being modeled. Currently the model only works when vertebrae T10-L3 are present in the dataset. Although this requirement is met for most abdominal CTs, we could improve the spine alignment to accommodate data sets with fewer vertebrae.

In conclusion, we have developed a novel SLM that provides accurate and fast initialization of multiple organs in abdominal CT segmentation. 


\section{References}

1. Kass, M., Witkin, A., Terzopoulos, D.: Snakes: Active Contour Models. International Journal of Computer Vision, 321-331 (1988)

2. Caselles, V., Kimmel, R., Sapiro, G.: Geodesic active contours. In: IEEE International Conference on Computer Vision, Cambridge, MA USA (1995)

3. Cootes, T.F., Taylor, C., Cooper, D., Graham, J.: Active shape models - their training and application. Computer Vision and Image Understanding 61, 38-59 (1995)

4. Park, H., Bland, P.H., Meyer, C.R.: Construction of an Abdominal Probabilistic Atlas and its Application in Segmentation. IEEE Trans. Med. Imag. 22(4), 483-492 (2003)

5. Okada, T., Yokota, K., Masatoshi, H., et al.: Construction of Hierarchical Multi-Organ Statistical Atlases and Their Application to Multi-Organ Segmentation from CT Images. In: Metaxas, D., Axel, L., Fichtinger, G., Székely, G. (eds.) MICCAI 2008, Part I. LNCS, vol. 5241, pp. 502-509. Springer, Heidelberg (2008)

6. Shimizu, A., Ohno, R., Ikegami, T., Kobatake, H., et al.: Segmentation of multiple organs in non-contrast 3D abdominal CT images. Int. J. CARS (2), 135-142 (2007)

7. Kaneko, T., Gu, L., Fujimoto, H.: Abdominal organ recognition using 3D mathematical morphology. In: Proc. of 15th Int Conf. on Pattern Recognition, Barcelona (2000)

8. Yao, J., O'Connor, S.D., Summers, R.M.: Automated Spinal Column Extraction and Partitioning. In: IEEE ISBI, Arlington, VA (2006) 